\title{
Plasma $n-3$ polyunsaturated fatty acids are negatively associated with obesity
}

\author{
Michelle Micallef ${ }^{1}$, Irene Munro ${ }^{1}$, Melinda Phang ${ }^{1}$ and Manohar Garg ${ }^{1,2 *}$ \\ ${ }^{1}$ Nutraceuticals Research Group, School of Biomedical Sciences, University of Newcastle, Callaghan, NSW 2308, Australia \\ ${ }^{2}$ Hunter Medical Research Institute, John Hunter Hospital, New Lambton, NSW 2310, Australia
}

(Received 3 October 2008 - Revised 19 March 2009 - Accepted 22 April 2009 - First published online 19 May 2009)

The objective of the present study was to investigate the relationship between plasma $n-3$ PUFA composition and weight status. A total of 124 adults, stratified by weight status: healthy weight $(n 21)$, overweight $(n$ 40) and obese $(n$ 63) were recruited. Fasting blood samples, anthropometric measures and body composition were collected. Plasma fatty acid composition was determined by GC. BMI, waist circumference and hip circumference were inversely correlated with $n-3$ PUFA, EPA and DHA $(P<0.05$ for all $)$ in the obese group. Obese individuals had significantly lower plasma concentrations of total $n$-3 PUFA, compared with healthy-weight individuals (4.53 (SD 1.11) v. $5 \cdot 25$ (SD 1.43 ) \%). When subjects were pooled and stratified into quartiles of total $n-3$ PUFA, a significant inverse trend was found for BMI $(P=0.002)$, waist circumference and hip circumference $(P=0.01$ and $P<0.001$ respectively). Higher plasma levels of total $n-3$ PUFA are associated with a healthier BMI, waist circumference and hip circumference. Our findings suggest that $n$-3 PUFA may play an important role in weight status and abdominal adiposity.

n-3 Fatty acids: Obesity: Lipids

Obesity is a consequence of the excessive accumulation of fat in adipose tissue which can result in significant morbidity and mortality. Health problems associated with obesity include cardiovascular disorders such as hypertension, stroke and $\mathrm{CHD}$, conditions associated with insulin resistance such as type 2 diabetes, and certain types of cancers ${ }^{(1,2)}$. A weight loss of between 5 and $10 \%$ can substantially reduce these risks ${ }^{(3,4)}$; however, successfully maintaining weight loss, in the long term, is difficult ${ }^{(5)}$. Hence, effective strategies to improve adherence to weight loss and weight maintenance are needed $^{(6)}$.

The consumption of $n$-3 PUFA, namely EPA and DHA, have been linked to reduced CVD risk $^{(7-9)}$, and to reduced fasting glucose levels, providing a protective effect against the development of type 2 diabetes ${ }^{(10)}$. There is also continuing debate as to whether or not $n$-3 PUFA contribute to weight loss.

Dietary fatty acids are an important source of adipose tissue fatty acids and play a significant role in adipose tissue metabolism $^{(11)}$. Intake of $n-3$ PUFA has been shown to influence the fatty acid composition of membrane phospholipids, thus modulating several metabolic processes that take place in the adipocyte ${ }^{(12-15)}$. Lipid management at the cellular level influences the degree of the development of disease and co-morbidities in obesity ${ }^{(16)}$. Indeed, abnormal $n$-3 PUFA metabolism in studies of obese children has been suggested ${ }^{(17-20)}$; therefore, not only the amount of dietary fat, but also the composition of dietary fat, plays an important role in adipose tissue metabolism and thus on body fat accumulation.

In the present study, we investigate the relationship between plasma $n-3$ PUFA concentration and various anthropometric measures in healthy-weight, overweight and obese adults. We hypothesise that plasma $n-3$ PUFA is associated with weight status, more specifically obesity. Perhaps $n-3$ PUFA could assist weight loss by complementing existing weightloss approaches through their influence on biomarkers of obesity ${ }^{(13,21,22)}$. We also examine whether the contribution of $n-3$ PUFA concentration to covariates of body composition is independent of weight status.

\section{Experimental methods}

Participants

A total of 124 male and female free-living participants, aged 18-70 years were recruited from the university campus and the general community of Newcastle, Australia. Exclusion criteria for participation were: diagnosed diabetes mellitus; liver or other endocrine dysfunction; evidence of CVD, including angina or hypolipidaemic medication; chronic inflammatory disease; consumption of fish oil supplements; consumption of more than two fatty fish meals per week; on a restricted diet; BMI $<20$ or $>40 \mathrm{~kg} / \mathrm{m}^{2}$; tobacco smoking. Further biochemical exclusion criteria included fasting glucose $>6.8 \mathrm{mmol} / \mathrm{l}$ (1225 mg/l).

The present study was conducted according to the guidelines laid down in the Declaration of Helsinki; all procedures involving human subjects were approved by the Human Research Ethics Committee of the University of Newcastle, Australia. Written informed consent was obtained from all subjects.

Abbreviation: FM, fat mass.

* Corresponding author: Professor Manohar Garg, fax +61 024921 2028, email manohar.garg@newcastle.edu.au 


\section{Anthropometry assessment}

All anthropometric measurements were made with participants wearing light clothing and no shoes. BMI was calculated as body mass in kilograms $(\mathrm{kg})$ divided by the square of height in meters $(\mathrm{m})$ to the nearest $0 \cdot 1\left(\mathrm{~kg} / \mathrm{m}^{2}\right)$ using a calibrated balance beam scale (PCS Measurement, NSW, Australia). Waist circumference was measured at the mid-point between the lowest rib and the top of the hipbone; the hip measurement was taken at the fullest point of the hip, as viewed from the side. The waist:hip ratio was calculated as waist girth in centimetres $(\mathrm{cm})$ divided by hip girth $(\mathrm{cm})$. Single-frequency bioelectrical impedance was used to determine fat mass (FM) and fat-free mass (Maltron International, Rayleigh, Essex, UK). Measurements were taken in the supine position following $\mathrm{a}>10 \mathrm{~h}$ fast with no physical activity or alcohol consumption $24 \mathrm{~h}$ before testing. Calculations determined percentage FM $((\mathrm{FM} / \text { body weight }) \times 100)^{(23)}$.

\section{Plasma fatty acid analyses}

Fasting $(>10 \mathrm{~h})$ blood samples were collected into tubes precoated with EDTA by venepuncture. Samples were prepared by centrifuging for $10 \mathrm{~min}$ at $3000 \mathrm{~g}$ at $4^{\circ} \mathrm{C}$. Plasma samples were collected and stored at $-80^{\circ} \mathrm{C}$ until further analysis.

The fatty acid composition of plasma lipids was determined according to a modification in the method of Lepage \& Roy $^{(24)}$, using an acetyl chloride methylation procedure. Fatty acid methyl esters were quantified using GC (Hewlett Packard 6890; Hewlett Packard, Palo Alto, CA, USA). The identity of each fatty acid peak was ascertained by comparison of the peak's retention time with the retention times of synthetic standards of known fatty acid composition (Nu Check Prep, Elysian, MN, USA). The relative amount of each fatty acid was quantified by integrating the area under the peak and dividing the result by the total area for all fatty acids. Concentrations of $n-3$ PUFA were calculated by summing the respective 18-22 carbon atom fatty acids (linolenic acid, EPA, docosapentaenoic acid and DHA). Fatty acid results are reported as percentage of total fatty acids.

\section{Statistical analysis}

Data are presented as mean values and standard deviations. Preliminary assumption testing was conducted to check for normality, linearity, outliers and homogeneity of variance, with no serious violations noted for anthropometric and body composition measurements. Variables that were not normally distributed were log-transformed before analysis. Comparisons between the different groups were made with one-way ANOVA and post hoc testing. $P<0.05$ was considered significant. Data were further explored with all weight-status groups pooled and stratified into quartiles of n-3 PUFA. All statistical analyses were carried out with SPSS software (version 15.0; SPSS Inc., Chicago, IL, USA).

\section{Results}

Among the 124 adults, the average age was 49.5 (SD 10.7) years, with $37 \%$ being male. Participants were stratified into weight status according to BMI (healthy weight $20-24.9 \mathrm{~kg} / \mathrm{m}^{2}$ (n 21), overweight $25-29 \cdot 9 \mathrm{~kg} / \mathrm{m}^{2}(n$ 40) and obese $30-40 \mathrm{~kg} / \mathrm{m}^{2}(n$ 63)). Anthropometric characteristics of the three groups are presented in Table 1. The healthy-weight group had a significantly lower body weight, BMI, waist circumference, hip circumference and FM $(P<0.001$ for all $)$ compared with the obese group. The overweight group had a significantly lower body weight, BMI, waist circumference, hip circumference $(P<0.001$ for all $)$ and FM $(P=0.03)$ compared with the obese group.

The correlation between plasma $n$-3 PUFA concentration and features of anthropometry were explored separately for each weight status. No significant correlation was observed for the healthy-weight and overweight groups (data not shown). Correlations between plasma $n$-3 PUFA concentration and BMI, waist circumference, hip circumference, waist:hip ratio and FM in the obese group were analysed (Table 2). Total n-3 PUFA, EPA and DHA were inversely correlated with BMI $(P=0.004, P=0.009, P=0.004$, respectively $)$, waist circumference $(P=0 \cdot 03, P=0 \cdot 05, P=0 \cdot 02$, respectively) and hip circumference $(P<0.001, \quad P=0.009, \quad P=0.002$, respectively).

When participants were stratified into quartiles according to total $n$-3 PUFA composition (thirty-one subjects per quartile (quartile 1: 3.4 (SD 0.06); quartile 2: 4.1 (SD 0.02); quartile 3: 4.9 (SD 0.06); quartile 4: 6.7 (SD 0.1)\% total fatty acids)), a highly significant inverse trend was found for BMI $(P=0 \cdot 002)$, waist circumference $(P=0.01)$ and hip circumference $(P<0.001)$ (Fig. 1). No trends were found for waist:hip

Table 1. Subject characteristics

(Mean values and standard deviations)

\begin{tabular}{|c|c|c|c|c|c|c|}
\hline & \multicolumn{2}{|c|}{$\begin{array}{l}\text { Healthy weight } \\
\qquad(n 21)\end{array}$} & \multicolumn{2}{|c|}{ Overweight ( $n$ 40) } & \multicolumn{2}{|c|}{ Obese $(n 63)$} \\
\hline & Mean & SD & Mean & SD & Mean & SD \\
\hline Age (years) & $55 \cdot 28^{a}$ & 8.56 & $49 \cdot 87^{a}$ & 11.46 & $43 \cdot 79^{b}$ & $12 \cdot 22$ \\
\hline Body weight (kg) & $66 \cdot 34^{a}$ & $9 \cdot 23$ & $80 \cdot 54^{b}$ & 8.08 & $95.53^{C}$ & 14.50 \\
\hline BMI $\left(\mathrm{kg} / \mathrm{m}^{2}\right)$ & $23 \cdot 12^{\mathrm{a}}$ & $1 \cdot 61$ & $27 \cdot 74^{b}$ & 1.56 & $33.59^{c}$ & $2 \cdot 72$ \\
\hline Waist $(\mathrm{cm})$ & $83 \cdot 07^{a}$ & $9 \cdot 37$ & $94.05^{b}$ & $7 \cdot 84$ & $104 \cdot 49^{c}$ & 8.86 \\
\hline Hip (cm) & $91 \cdot 36^{a}$ & $6 \cdot 45$ & $104 \cdot 31^{b}$ & $8 \cdot 18$ & $118 \cdot 63^{c}$ & 8.94 \\
\hline Waist:hip ratio & 0.91 & 0.09 & 0.90 & 0.08 & 0.98 & 0.08 \\
\hline Fat mass (\%) & $33 \cdot 81^{a}$ & $7 \cdot 37$ & $37 \cdot 36^{a}$ & $7 \cdot 38$ & $40 \cdot 90^{b}$ & $6 \cdot 70$ \\
\hline Fat-free mass (\%) & $66 \cdot 57^{a}$ & 8.07 & $62 \cdot 89^{a}$ & $7 \cdot 90$ & $59 \cdot 10^{b}$ & $6 \cdot 70$ \\
\hline
\end{tabular}

${ }^{a, b, c}$ Mean values within a row with unlike superscript letters were significantly different $(P<0.05)$. 
Table 2. Associations between plasma $n-3$ PUFA (\% of total fatty acids) and measures of anthropometry in obese subjects

(Pearson's correlations)

\begin{tabular}{lcccr}
\hline & Total $n-3$ PUFA & Linolenic acid & EPA & DHA \\
\hline BMI $\left(\mathrm{kg} / \mathrm{m}^{2}\right)$ & $-0.40^{*}$ & -0.11 & $-0.32^{*}$ & $-0.36^{\star}$ \\
Waist $(\mathrm{cm})$ & $-0.27^{\star}$ & -0.01 & $-0.24^{*}$ & $-0.28^{*}$ \\
Hip $(\mathrm{cm})$ & $-0.41^{*}$ & -0.07 & $-0.32^{*}$ & $-0.38^{*}$ \\
Waist:hip ratio & 0.17 & 0.12 & 0.10 & 0.11 \\
Fat mass (\%) & -0.03 & -0.12 & 0.14 & -0.16 \\
\hline
\end{tabular}

${ }^{*} P<0.05$

ratio $(P=0 \cdot 50)$ and FM $(P=0 \cdot 16)$. Post hoc analysis shows a significant difference between the lowest and highest quartiles for BMI $(P=0.004)$, waist circumference $(P=0.03)$ and hip circumference $(P=0.001)$.

\section{Discussion}

In the present cross-sectional study, we observed significantly lower plasma concentrations of $n-3$ PUFA in obese men and women compared with healthy-weight individuals. The finding of the present study is that measures of weight status were correlated with plasma $n-3$ PUFA composition when participants were stratified into quartiles of total n-3 PUFA concentration.

Similar findings were reported in a study comparing serum phospholipid fatty acids with adipose tissue in twenty-five normal-weight and obese adolescents, which found that concentrations of $n-3$ PUFA were significantly lower in obese $v$. lean, age-matched, females ${ }^{(17)}$. Another study involving 120 normal-weight and overweight adolescents found that overweight adolescents had lower total $n-3$ PUFA and lower DHA concentrations compared with normal-weight adolescents, independent of body fat and fat distribution ${ }^{(25)}$. When dietary intake was also considered in a study of 134 age- and sex-matched normal-weight and overweight children, the BMI $z$-score of the obese children was negatively associated with plasma $n-3$ PUFA and DHA, despite obese children having higher intakes of the main fatty acid families, including PUFA ${ }^{(26)}$.

Previous observational studies which also considered food intake indicate a negative association of fish consumption with central obesity measures ${ }^{(27)}$. In rodents, feeding fish oilenriched diets have been shown to prevent abdominal fat accumulation compared with other types of dietary oils ${ }^{(28-30)}$. In human subjects, replacement of $6 \mathrm{~g}$ visible fat/d with $6 \mathrm{~g}$ fish oil/d for 3 weeks resulted in reduced fat mass and increased basal lipid oxidation ${ }^{(31)}$. A recent study has shown that the inclusion of lean fish, fatty fish or fish oil to a nutritionally balanced diet resulted in a greater weight loss within 4 weeks compared with diets devoid of seafood or marine supplements $^{(32)}$. Another study which investigated the dietary intake of 132 children aged 4 years reported that a low $n-3$ PUFA intake was associated with higher body weight ${ }^{(33)}$. These studies, along with our observations, suggest that $n-3$ PUFA supplementation may play an important role in preventing weight gain and improving weight loss when $n$-3 PUFA are supplemented concomitantly with a structured weight-loss programme. Furthermore, inclusion of $n-3$ PUFA in a weight-loss programme may provide additional health benefits ${ }^{(8)}$.
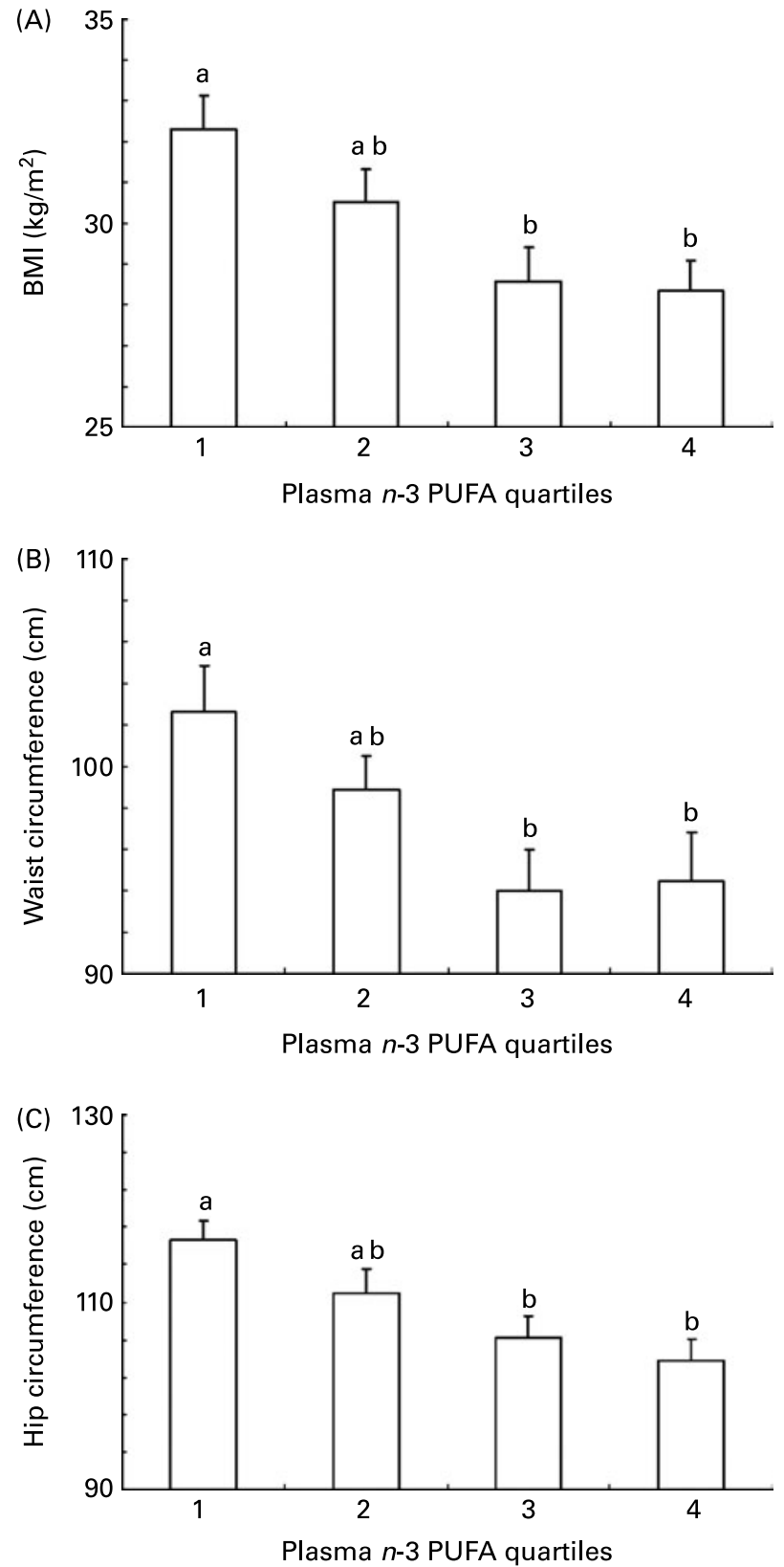

Fig. 1. Quartiles of plasma $n-3$ PUFA concentration for (A) BMI $\left(\mathrm{kg} / \mathrm{m}^{2}\right)$, $(B)$ waist circumference $(\mathrm{cm})$ and $(C)$ hip circumference $(\mathrm{cm})$. Values are means ( $n 31$ per quartile), with standard deviations represented by vertical bars. ${ }^{\mathrm{a}, \mathrm{b}}$ Mean values with unlike letters were significantly different $(P<0.05)$. For BMI, $P$ for trend $=0.002$; for waist circumference, $P$ for trend $=0.01$; for hip circumference, $P$ for trend $<0.001$.

The results presented are biologically plausible because several mechanisms underlying the association between $n-3$ PUFA and obesity have been shown. One possibility is that $n$-3 PUFA could increase basal fat oxidation which may in turn reduce fat mass ${ }^{(9,31)}$. Animal studies have shown that $n$-3 PUFA supplementation may be associated with increased expression of mitochondrial uncoupling protein ${ }^{(34)}$, a system of thermogenesis that can provide a defence against obesity. Furthermore, a recent study has shown that $n$-3 PUFA intake increases postprandial satiety in overweight and obese individuals during weight $\operatorname{loss}^{(35)}$. Fatty acids may interact with 
neuroendocrine factors including insulin ${ }^{(36,37)}$, $\operatorname{ghrelin}^{(38,39)}$ and leptin ${ }^{(40-42)}$ to modulate brain-intestinal loop signals for energy metabolism and appetite control. A recent study found that ghrelin is negatively correlated with body weight and total $n$-3 PUFA in normal-weight subjects ${ }^{(43)}$, suggesting that $n-3$ PUFA can modulate appetite. Thus, the idea that fish oil can regulate weight status via improved appetite control along with a subsequent reduction in energy intake is plausible and worthy of further investigation.

A limitation of our study is that it does not explain why plasma $n$-3 PUFA concentration was lower in obese individuals. A possible reason could be that the diets of obese adults are such that their intake of $n-3$ PUFA (marine foods) is lower than in normal-weight individuals. Alternatively, lower plasma $n$-3 PUFA levels in obese individuals may be a reflection of increased utilisation or oxidative damage to these highly unsaturated fatty acids. Indeed, obesity has been associated with increased oxidative stress ${ }^{(44)}$. Future studies should also examine long-term biomarkers of $n-3$ PUFA status, such as the $n-3$ index, to further explore the relationship with obesity. We also acknowledge the small sample size of the present study, and recognise that a largescale multicentre trial would be appropriate; certainly future studies should account for ethnicity and family history of disease and obesity.

Regardless of the mechanisms by which $n-3$ PUFA may assist in the maintenance of weight status ${ }^{(16,35)}$, a significant inverse trend for BMI, waist circumference and hip circumference was observed when participants were stratified into quartiles of plasma $n-3$ PUFA concentration. Whether improvements in plasma concentration of $n-3$ PUFA by dietary supplementation with marine oils may reduce abdominal adiposity, or obesity in general, merits investigation. Interestingly, anthropometric measures correlated with the major $n-3$ PUFA (EPA and DHA) but not the parent $n$-3 PUFA (linolenic acid).

In summary, we have reported an inverse relationship between plasma concentrations of $n-3$ PUFA and anthropometric measures of obesity including BMI, waist circumference and hip circumference. Previous studies involving children and adolescents have shown a negative correlation between adiposity and plasma $n$-3 PUFA and DHA concentrations, but there appears to be a paucity of research in adults. These studies make the basis for conducting more intervention trials in adults examining the influence of dietary supplementation with $n-3$ PUFA-rich fats/oils in assisting weight loss and weight maintenance.

\section{Acknowledgements}

M. M. participated in the conception and design of the study, data collection and performed the statistical analysis and drafting of the manuscript. I. M. participated in the conception and design of the study, data collection and in drafting the manuscript. M. P. analysed the plasma fatty acid composition and was involved in drafting the manuscript. M. G. was involved in the coordination of the study, provided significant advice and consultation and participated in drafting the manuscript.

The authors have no conflict of interest to disclose.

\section{References}

1. World Health Organization (1998) Obesity: Preventing and Managing the Global Epidemic. Geneva: WHO.

2. World Health Organization (2003) Diet, Nutrition and the Prevention of Chronic Diseases. Joint WHO/FAO Expert Consultation. WHO Technical Report Series no. 916. Geneva: WHO.

3. Goldstein DJ (1992) Beneficial health effects of modest weight loss. Int J Obes Relat Metab Disord 16, 397-415.

4. Blackburn G (1995) Effect of degree of weight loss on health benefits. Obes Res 3, Suppl. 2, 211s-216s.

5. Hill JO, Thompson H \& Wyatt H (2005) Weight maintenance: what's missing? J Am Diet Assoc 105, Suppl. 1, S63-S66.

6. Dansinger ML, Gleason JA, Griffith JL, et al. (2005) Comparison of the Atkins, Ornish, Weight Watchers, and Zone diets for weight loss and heart disease risk reduction: a randomized trial. JAMA 293, 43-53.

7. Kris-Etherton PM, Harris WS \& Appel LJ (2003) Fish consumption, fish oil, omega-3 fatty acids, and cardiovascular disease. Arterioscler Thromb Vasc Biol 23, e20-e30.

8. Krebs JD, Browning LM, McLean NK, et al. (2006) Additive benefits of long-chain $n-3$ polyunsaturated fatty acids and weight-loss in the management of cardiovascular disease risk in overweight hyperinsulinaemic women. Int J Obes (Lond) 30, 1535-1544.

9. Kunesova M, Braunerova R, Hlavaty P, et al. (2006) The influence of $n-3$ polyunsaturated fatty acids and very low calorie diet during a short-term weight reducing regimen on weight loss and serum fatty acid composition in severely obese women. Physiol Res 55, 63-72.

10. Friedberg CE, Janssen MJ, Heine RJ, et al. (1998) Fish oil and glycemic control in diabetes. A meta-analysis. Diabetes Care 21, 494-500.

11. Fernandez-Quintela A, Churruca I \& Portillo MP (2007) The role of dietary fat in adipose tissue metabolism. Public Health Nutr 10, 1126-1131.

12. Hajer GR, van Haeften TW \& Visseren FL (2008) Adipose tissue dysfunction in obesity, diabetes, and vascular diseases. Eur Heart J 29, 2959-2971.

13. Lopez-Garcia E, Schulze MB, Manson JE, et al. (2004) Consumption of $(n-3)$ fatty acids is related to plasma biomarkers of inflammation and endothelial activation in women. $J$ Nutr 134, 1806-1811.

14. Flachs P, Mohamed-Ali V, Horakova O, et al. (2006) Polyunsaturated fatty acids of marine origin induce adiponectin in mice fed a high-fat diet. Diabetologia 49, 394-397.

15. von Schacky C (2007) n-3 PUFA in CVD: influence of cytokine polymorphism. Proc Nutr Soc 66, 166-170.

16. Cave MC, Hurt RT, Frazier TH, et al. (2008) Obesity, inflammation, and the potential application of pharmaconutrition. Nutr Clin Pract 23, 16-34.

17. Karlsson M, Marild S, Brandberg J, et al. (2006) Serum phospholipid fatty acids, adipose tissue, and metabolic markers in obese adolescents. Obesity (Silver Spring) 14, 1931-1939.

18. Ailhaud G \& Guesnet P (2004) Fatty acid composition of fats is an early determinant of childhood obesity: a short review and an opinion. Obes Rev 5, 21-26.

19. Decsi T, Molnar D \& Koletzko B (1996) Long-chain polyunsaturated fatty acids in plasma lipids of obese children. Lipids $\mathbf{3 1}$, 305-311.

20. Agostoni C, Riva E, Bellu R, et al. (1994) Relationships between the fatty acid status and insulinemic indexes in obese children. Prostaglandins Leukot Essent Fatty Acids 51, 317-321.

21. Simopoulos AP (2002) Omega-3 fatty acids in inflammation and autoimmune diseases. $J$ Am Coll Nutr 21, 495-505.

22. Yaqoob P (2004) Fatty acids and the immune system: from basic science to clinical applications. Proc Nutr Soc 63, 89-104. 
23. Kyle UG, Bosaeus I, De Lorenzo AD, et al. (2004) Bioelectrical impedance analysis - part I: review of principles and methods. Clin Nutr 23, 1226-1243.

24. Lepage G \& Roy CC (1986) Direct transesterification of all classes of lipids in a one-step reaction. J Lipid Res 27, 114-120.

25. Klein-Platat C, Drai J, Oujaa M, et al. (2005) Plasma fatty acid composition is associated with the metabolic syndrome and lowgrade inflammation in overweight adolescents. Am J Clin Nutr 82, $1178-1184$.

26. Scaglioni S, Verduci E, Salvioni M, et al. (2006) Plasma longchain fatty acids and the degree of obesity in Italian children. Acta Paediatr 95, 964-969.

27. Ghosh A, Bose K \& Das Chaudhuri AB (2003) Association of food patterns, central obesity measures and metabolic risk factors for coronary heart disease (CHD) in middle aged Bengalee Hindu men, Calcutta, India. Asia Pac J Clin Nutr 12, 166-171.

28. Belzung F, Raclot $\mathrm{T} \&$ Groscolas R (1993) Fish oil $n-3$ fatty acids selectively limit the hypertrophy of abdominal fat depots in growing rats fed high-fat diets. Am J Physiol 264, R1111-R1118.

29. Ikemoto S, Takahashi M, Tsunoda N, et al. (1996) High-fat diet-induced hyperglycemia and obesity in mice: differential effects of dietary oils. Metabolism 45, 1539-1546.

30. Hill JO, Peters JC, Lin D, et al. (1993) Lipid accumulation and body fat distribution is influenced by type of dietary fat fed to rats. Int J Obes Relat Metab Disord 17, 223-236.

31. Couet C, Delarue J, Ritz P, et al. (1997) Effect of dietary fish oil on body fat mass and basal fat oxidation in healthy adults. Int $J$ Obes Relat Metab Disord 21, 637-643.

32. Thorsdottir I, Tomasson H, Gunnarsdottir I, et al. (2007) Randomized trial of weight-loss-diets for young adults varying in fish and fish oil content. Int J Obes (Lond) 31, 1560-1566.

33. Garemo M, Lenner RA \& Strandvik B (2007) Swedish pre-school children eat too much junk food and sucrose. Acta Paediatr 96, 266-272.
34. Katsumi I, Masahiro O, Mayumi S, et al. (2002) Effects of fish oil feeding on obesity and UCP expression in dogs. Vet Biochem 39, $31-38$.

35. Parra D, Ramel A, Bandarra N, et al. (2008) A diet rich in long chain omega-3 fatty acids modulates satiety in overweight and obese volunteers during weight loss. Appetite 51, 676-680.

36. Nettleton JA \& Katz R (2005) n-3 Long-chain polyunsaturated fatty acids in type 2 diabetes: a review. J Am Diet Assoc 105, 428-440.

37. Haugaard SB, Madsbad S, Hoy CE, et al. (2006) Dietary intervention increases $n$-3 long-chain polyunsaturated fatty acids in skeletal muscle membrane phospholipids of obese subjects. Implications for insulin sensitivity. Clin Endocrinol (Oxf) 64, 169-178.

38. Murphy KG, Dhillo WS \& Bloom SR (2006) Gut peptides in the regulation of food intake and energy homeostasis. Endocr Rev 27, 719-727.

39. Cummings DE \& Overduin J (2007) Gastrointestinal regulation of food intake. J Clin Invest 117, 13-23.

40. Winnicki M, Somers VK, Accurso V, et al. (2002) Fish-rich diet, leptin, and body mass. Circulation 106, 289-291.

41. Perez-Matute P, Marti A, Martinez JA, et al. (2007) Conjugated linoleic acid inhibits glucose metabolism, leptin and adiponectin secretion in primary cultured rat adipocytes. Mol Cell Endocrinol 268, 50-58.

42. Mori TA, Burke V, Puddey IB, et al. (2004) Effect of fish diets and weight loss on serum leptin concentration in overweight, treated-hypertensive subjects. J Hypertens 22, 1983-1990.

43. Barber-Heidal K, Broughton K, Malinauskas B, et al. (2008) Relationships among omega-3 fatty acids, anthropometrics, and biochemical markers in college students. FASEB $J \mathbf{2 2}$, 10933.

44. Furukawa S, Fujita T, Shimabukuro M, et al. (2004) Increased oxidative stress in obesity and its impact on metabolic syndrome. J Clin Invest 114, 1752-1761. 